KOMPASS

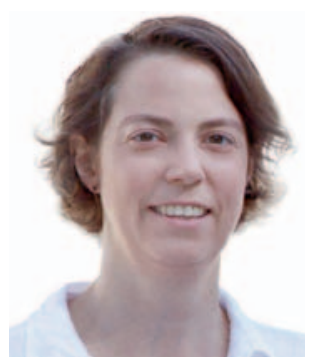

Dr. Ute Mons

Deutsches Krebsforschungszentrum, Heidelberg, Deutschland

Das Jahr 2016 bescherte der deutschen Tabakkontrolle einige bemerkenswerte Erfolge. Mit dem Inkrafttreten der EUTabakproduktrichtlinie wurden die Zigarettenhersteller zum Abdruck großer bildgestützter Warnhinweise («Schockbilder») verpflichtet und elektronische Zigaretten (E-Zigaretten) erstmals gesetzlich reguliert; darüber hinaus wurde ein Abgabeverbot von E-Zigaretten an Minderjährige eingeführt. Das Jahr 2016 hielt aber auch eine schwere Enttäuschung bereit: Ein bereits vom Kabinett beschlossener Gesetzentwurf zur Einführung des längst überfälligen Außenwerbeverbots für Tabakprodukte Deutschland ist das einzige EU-Land, das noch uneingeschränkt Außenwerbung für Tabakprodukte erlaubt - wird bis heute von Teilen der CDU/CSU-Fraktion blockiert. Dabei hatte sich Deutschland bereits im Jahr 2004 mit der Unterzeichnung und Ratifizierung der WHO-Tabakrahmenkonvention dazu verpflichtet, innerhalb von fünf Jahren nach Inkrafttreten - also bis 2010 - ein umfassendes Tabakwerbeverbot einzuführen. Die Umsetzung dieser Verpflichtung wird nun weiterhin verschleppt, obwohl knapp drei Viertel der Bevölkerung ein Außenwerbeverbot unterstützen [1] Schon mehrfach wurde in Deutschland um Tabakwerbeverbote gerungen. Erstmals im Jahr 1974, als Tabakwerbung in Rundfunk

\section{Literatur}

1 Schaller K, Mons U: Große Zustimmung zu einem Verbot der Tabakaußenwerbung. Aus der Wissenschaft - für die Politik. Heidelberg, Deutsches Krebsforschungszentrum, 2016.

2 Deutscher Bundestag: 108. Sitzung. Bonn, 18 Juni 1974. http://dipbt.bundestag.de/doc/btp/ 07/07108.pdf (Zugriff 25.01.2017)

\title{
Tabakpräventionspolitik in Deutschland: Nachholbedarf trotz einiger Fortschritte
}

und Fernsehen verboten werden sollte. «Die Werbung ist ein Informations- und [...] kein Manipulationsinstrument. Durch eine Einschränkung der Werbung wird man keinen Jugendlichen vom Rauchen abhalten [...]. Persönlich allerdings bin ich der Meinung, daß gute Beispiele viel mehr bewirken», argumentierte damals der CDU-Abgeordnete Dr. Hammans - glücklicherweise erfolglos - gegen das Vorhaben [2]. Ganz ähnliche Einwände kommen heute aus dem Wirtschaftsflügel der CDU/CSU-Fraktion: Werbung sei ein legitimes Informationsinstrument und Aufklärung wirksamer als Verbotspolitik. Dass trotz eines immensen Zuwachses an wissenschaftlicher Evidenz für die Schädlichkeit des Rauchens bis heute die gleichen Gegenargumente vorgebracht werden, ist der massiven Lobbyarbeit der Tabakindustrie zu verdanken. Dabei ist eindeutig, dass die großflächige Plakatwerbung nicht etwa sachliche Informationen über Zigaretten verbreitet (Zigaretten machen abhängig, krank und töten jeden zweiten Konsumenten), sondern das Rauchen vielmehr so attraktiv wie möglich darstellt, indem sie es mit erstrebenswerten Attributen wie Unabhängigkeit, Freiheit und Jugendlichkeit verknüpft. Mit Aufklärung allein lässt sich dagegen nur wenig bewirken, solange für Aufklärungsmaßnahmen gegen das Rauchen jährlich lediglich

3 Schaller K, Mons U: Verbot der Außenwerbung für Tabakprodukte - Positionen und Fakten. Aus der Wissenschaft - für die Politik. Heidelberg, Deutsches Krebsforschungszentrum, 2016. etwa 2 Millionen EUR zur Verfügung stehen, die Tabakindustrie aber rund 200 Millionen Euro jährlich in Marketingmaßnahmen (darunter etwa 70 Millionen EUR allein für Außenwerbung) investiert [3].

Länder wie Australien zeigen, wie ein erfolgreicher Kampf gegen das Rauchen aussehen kann: eine konsequente Präventionspolitik mit einem Bündel wirksamer Maßnahmen wie regelmäßige Tabaksteuererhöhungen, einem umfassenden Nichtraucherschutz und weitreichenden Werbeverboten (bis hin zur Einheitsverpackung) hat dort zu einem Rückgang der Raucherprävalenz auf unter $10 \%$ und zu einer stetig sinkenden Lungenkrebsinzidenz geführt. Deutschland hinkt einer solchen Politik weit hinterher und ist in Europa Schlusslicht in Sachen Tabakprävention. Angesichts von jährlich über 120000 Todesfällen, die auf das Rauchen zurückzuführen sind Tendenz steigend [4] - muss sich die Politik endlich zu wirksamen Maßnahmen gegen das Rauchen durchringen und konsequent Gesundheitsinteressen über wirtschaftliche Interessen der Tabakindustrie stellen.

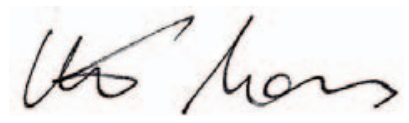

Dr. Ute Mons
4 Mons U, Brenner H: Demographic ageing and the evolution of smoking-attributable mortality: the example of Germany. Tobacco Control DOI:10.1136/tobaccocontrol-2016-053008.

\section{KARGER}

() 2017 S. Karger GmbH, Freiburg

Fax +497614520714

information@karger.com

www.karger.com
Accessible online at: www.karger.com/kkp
Dr. Ute Mons

Deutsches Krebsforschungszentrum,

Stabsstelle Krebsprävention und WHO-Kollaborationszentrum für Tabakkontrolle

Im Neuenheimer Feld 280, 69120 Heidelberg, Deutschland

u.mons@dkfz.de 


\section{FOKUS}

\section{Nikotinsucht und Lungenschäden durch Rauch}

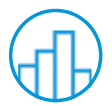

\section{Epidemiologie}

Rauchen ist die häufigste vermeidbare Todesursache in den Industrieländern. Dank vereinten Anstrengungen, Werbeverboten und Preissteigerung der Zigaretten geht der Raucheranteil unter Kindern, Jugendlichen und Erwachsenen zumindest in Deutschland kontinuierlich leicht zurück [1, 2]. Bis sich dieser Rückgang jedoch in einer sinkenden Sterblichkeitsrate niederschlägt, wird es noch einige Jahrzehnte dauern, da sich die rauchbedingten Schäden oft erst im höheren Alter bemerkbar machen. Ca. 25\% der deutschen Bevölkerung rauchen regelmäßig - dies entspricht im internationalen Vergleich höchstens dem Durchschnitt. Die Folgen: Im Jahr 2013 waren ca. 13,5\% aller Todesfälle in Deutschland mit dem Rauchen assoziiert [2] Rauchen - aktiv wie passiv - verursacht verschiedene Krebsarten, wobei die Lunge besonders betroffen ist: Rund $80 \%$ aller Lungenkrebsfälle sind auf das Rauchen zurückzuführen. Der blaue Dunst ist auch die bedeutendste Ursache für die chronisch-obstruktive Lungenerkrankung (COPD), erhöht das Risiko von Tuberkulose, Herz-KreislaufErkrankungen und Schlaganfällen um ein Vielfaches, fördert akute Erkrankungen der Atemwege wie Grippe und Erkältungen und schädigt das Ungeborene in der Schwangerschaft nachhaltig [2].

\section{Herausforderungen und Handlungsbedarf}

In den letzten Jahren fand ein wichtiges Umdenken statt - Die Tabakabhängigkeit wird nicht mehr nur als «ungesunde Lebensgewohnheit», sondern als «krankheit» betrachtet. Erst dies ermöglichte, dass die Therapie der Tabakabhängigkeit unter bestimmten Bedingungen (starke oder sehr starke Nikotinabhängigkeit gemäß FagerströmFragebogen und/oder Präsenz von rauchbedingten Folgekrankheiten) beispielsweise in der Schweiz auch von der Krankenkasse übernommen wird. So hatte es Champix ${ }^{\complement}$ per 1. Juli 2013 auf die Spezialitätenliste geschafft $[5,6]$. Gleichzeitig wächst das Interesse am «Dampfen», insbesondere unter Jugendlichen und jungen Erwachsenen, die bislang noch nie geraucht hatten. Gerade bei dieser vulnerablen Gruppe wird diskutiert, ob das Dampfen den Einstieg zum Tabakrauchen erleichtern könnte. Beim Dampfen werden unterschiedliche Substanzen mit und ohne Zugabe von Nikotin inhaliert - bei vielen ist bisher die Unbedenklichkeit nicht gezeigt worden. Immer wieder wird über Nebenwirkungen berichtet, sodass die E-Zigarette aktuell nicht für den Rauchstopp empfohlen werden kann. Es ist zudem nicht ermutigend, dass die internationale Tabakindustrie zur den wichtigsten Promotoren der E-Zigarette gehört. Immerhin ist in Deutschland seit April 2016 der Verkauf von E-Zigaretten an Kinder und Jugendliche verboten.

\section{Therapieoptionen}

Etwa die Hälfte der Raucher schafft es im Laufe des Lebens, mit dem Rauchen dauerhaft aufzuhören: sei es aus medizinischen oder sozialen Gründen. Erfreulicherweise gelingt 76\% der Raucher der Ausstieg ohne jegliche Unterstützung. Das Aufhören zahlt sich bereits früh aus: Schon nach wenigen Tagen verbessern sich die Atemwegsfunktionen und der Blutdruck sinkt. Innerhalb weniger Jahre verringert sich das Erkrankungsrisiko für Herz-Kreislauferkrankungen und die meisten Krebsarten. Selbst bei über 60-Jährigen verzögert ein Rauchstopp das Risiko, zu sterben, im Vergleich zu denjenigen, die weiterrauchen, um mehrere Jahre [2].

Als erfolgreichste Unterstützung bei einem Rauchstopp hat sich eine Verhaltenstherapie, ergänzt durch Nikotinersatzprodukte und Champix ${ }^{\odot}$, erwiesen. Die Rolle der E-Zigarette zur Unterstützung des Rauchstopps ist derzeit nicht geklärt und kann derzeit nicht empfohlen werden, wird aber in ca. 6\% als Hilfsmittel im Individualfall eingesetzt. Raucher, die den Ausstieg selber oder unterstützt durch den Hausarzt nicht schaffen, sollten eine Spezialambulanz für den assistierten Rauchstopp aufsuchen.

\section{Literatur}

1 Deutsche Krebsgesellschaft: Rauchen. Zahlen und Fakten. www.krebsgesellschaft.de/onko-internetportal/basis-informationen-krebs/bewusst-leben/ rauchen-zahlen-und-fakten.html (Zugriff 29.12.16).

2 Deutsches Krebsforschungszentrum: Tabakatlas Deutschland 2015. Heidelberg, DKFZ, 2015.

3 Statistisches Bundesamt: Absatz von Zigaretten im 3. Quartal 2016 rückläufig. Pressemitteilung Nr. 374 vom 18.10.2016. www.destatis.de/DE/ PresseService/Presse/Pressemitteilungen/2016/10/PD16_374_799.html (Zugriff 29.12.16).

4 Ehrenstein C: In welchen Berufen am meisten geraucht wird. www.welt de/politik/deutschland/article148400974/In-welchen-Berufen-am-meistengeraucht-wird.html (Zugriff 29.12.16).

5 Schweizerische Eidgenossenschaft: Die obligatorische Krankenpflegeversicherung vergütet künftig das Raucherentwöhnungsmittel Champix. Medienmitteilung vom 01.07.2013. www.admin.ch/gov/de/start/dokumentation/medienmitteilungen.msg-id-49514.html (Zugriff 02.01.2017).

6 Raucherentwöhnungsmittel: FDA stuft Warnung zurück. Pharmazeutische Zeitung Online, 19.12.2016. www.pharmazeutische-zeitung.de/index. php?id=66757 (Zugriff 02.01.2017)

Prof. Dr. Martin Brutsche, Chefarzt, Kantonsspital St.Gallen, Bereich Pneumologie und interdisziplinäres Zentrum für Schlafmedizin 